\title{
Experimental Performance Evaluation of BLE 4 vs BLE 5 in Indoors and Outdoors Scenarios
}

\author{
Heikki Karvonen, Konstantin Mikhaylov, \\ Matti Hämäläinen, Jari Iinatti \\ Centre for Wireless Communications \\ University of Oulu \\ Finland \\ \{heikki.karvonen, konstantin.mikhaylov, \\ matti.hamalainen, jari.iinatti\}@ oulu.fi
}

\author{
Carlos Pomalaza-Ráez \\ Department of Electrical and Computer Engineering, \\ Purdue University \\ Fort Wayne, Indiana, USA \\ cpomalaz@purdue.edu
}

\begin{abstract}
This paper focus on an experimental performance evaluation of the recently published Bluetooth Low Energy (BLE) 5 technology. Measurements have been conducted both in indoors and outdoors scenarios. Performance of BLE 5 is compared to a previous release of BLE 4 which is currently the most used technology in commercial wireless healthcare and medical devices. This new improved BLE version may continue fostering the success of BLE use in those application scenarios as well as enable novel Internet of Things solutions. The main goal of this work was to evaluate, experimentally, the communications range and throughput performance of BLE 5 coded version which claims to provide 4fold improvement to the previous version of BLE. Measurement results obtained using the Nordic Semiconductor nRF52840 chipset are reported for indoor and outdoor cases relevant to healthcare and medical scenarios. Results show the practical communications range and throughput of the BLE 5 coded version, giving insight about the possible application space improvements for BLE technology. Specifically, our measurements showed that BLE 5 coded mode provides approximately $9 \mathrm{~dB}$ radio link budget gain compared to BLE 4, which leads to more than 2-fold communications range improvement in line-of-sight outdoor scenario and $10-20 \%$ improvement in non-line-of-sight indoor scenario.
\end{abstract}

\section{CCS CONCEPTS}

- Networks $\rightarrow$ Physical links; Network measurement; Network range; Short-range networks; Ad hoc networks; Network reliability - Computer systems organization $\rightarrow$ Sensor networks;

Permission to make digital or hard copies of all or part of this work for personal or classroom use is granted without fee provided that copies are not made or distributed for profit or commercial advantage and that copies bear this notice and the full citation on the first page. Copyrights for components of this work owned by others than ACM must be honored. Abstracting with credit is permitted. To copy otherwise, or republish, to post on servers or to redistribute to lists, requires prior specific permission and/or a fee. Request permissions from permissions@acm.org.

BODYNETS'17, September 2017, Dalian, People's Republic of China

(C) 2017 ACM. 123-4567-24-567/08/06 . . \$15.00

DOI: $10.475 / 123 \quad 4$

\section{KEYWORDS}

BLE 5.0, communications range, unlicensed band, healthcare and medical applications, Internet of Things.

\section{INTRODUCTION}

Over recent years, the use of wireless body area networks (WBAN) has been continuously increasing [1], [2]. Today these devices are used in the context of smart healthcare applications, operating in hospitals and other similar environments, as well as for versatile sport and fitness activities. Due to the very nature of these use cases, wireless links are the most efficient way to enable connection of WBAN devices between themselves as well as to the external world and backbone systems.

Among all wireless standards employed for medical applications, Bluetooth Low Energy (BLE) stands out as the most widely used in current commercially available products [3]. The range of today's products communicating using BLE spans from simple pulse oximeters to complete body networks measuring the wide sets of physical parameters, such as body temperature, electrocardiogram (ECG) and electroencephalogram (EEG). BLE operates in the industrial, scientific and medical (ISM) $2.4 \mathrm{GHz}$ band, which is available worldwide enabling interoperability in different regions. Nonetheless, today this band is rapidly becoming congested due to the presence of several other wireless technologies such as IEEE Std. 802.11x (Wi-Fi) [4], IEEE Std. 802.15.4 [5] (ZigBee [6]), IEEE Std. 802.15.6 [7], ETSI SmartBAN [8], ANT / ANT+ [9] and most recently, the upcoming unlicensed LTE solutions (LTE-U) [10]. Despite the competition for the spectrum and the corresponding interference and coexistence issues [11], Bluetooth has shown a considerable resilience and continuous improvements since it was introduced already in the late 1990s.

The low-power version of Bluetooth, BLE 4, has been in use since June 2010 and today it can be found in almost every smartphone, tablet, and laptop in the market in addition to a large set of other wireless devices. The most recent version, Bluetooth 5 was introduced in December 2016 [12] with the first commercial development kits being available in early 2017. Upcoming state-ofthe-art smartphones are expected to support Bluetooth 5. The first 
smartphone supporting Bluetooth 5 is Samsung Galaxy S8, which is however lacking the support for the long-range LE coded mode. The long-range feature has made BLE 5 very suitable for versatile Internet of Things (IoT) applications. The official announcement of BLE 5 states that the increase in range is up to 4 times compared with BLE 4.2 [13].

To the best of our knowledge, the currently available literature doesn't provide results of any experimental investigation of the BLE 5 performance. In this paper we aim at fixing this omission by reporting the results of a comprehensive experimental measurement campaign, studying the actual range and throughput of BLE 5 in real indoor and outdoor scenarios. The measurements are conducted using one of the first available BLE 5 commercial chipsets. Importantly, we conduct our measurements and report the results not for BLE 5 only, but also for BLE 4.2. The latter is used as the reference, enabling the mapping of our results against the ones of previous works and allowing to easier estimate the benefits of using BLE 5 in an application currently employing BLE 4.2. Another contribution of this paper, which is especially important in the context of medical / health care WBANs, is that in our experiments we also address the cases of multi-floor buildings and outdoor scenarios, implying the cases when mobile patients might want to spend time such places as gardens and well delineated sidewalks.

The structure of the rest of the paper is as follows. Section 2 briefly describes the specifics of the BLE 5 technology. Section 3 details the hardware and software used in our measurements, Section 4 and Section 5 details the indoor and outdoor measurements, respectively. Section 6 provides a discussion of the results. Conclusions and future work can be found in Section 7.

\section{FEATURES OF THE BLE 5 TECHNOLOGY}

The BLE 5 represents a further evolution of the BLE technology, which comprehensively addresses the major problems of its predecessor, namely the limited range, long transmission times and limited functionality of the broadcasting modes. In the rest of this section we will focus on the most important changes of BLE 5 compared to BLE 4.2.

The problem of improving the communications range and the maximum throughput has been addressed in BLE 5 specification by introducing three new physical layer (PHY) options. In addition to the $1 \mathrm{Mbit} / \mathrm{s}$ Gaussian frequency shift keying (GFSK) of BLE 4 (addressed in Bluetooth $\vee 5.0$ core specification as LE 1M), the BLE 5 specifies a $2 \mathrm{Mbit} / \mathrm{s}$ GFSK PHY (named $L E 2 M$ ) for short range high-speed transmission and two coded PHY (referred to as $L E$ Coded) with payload coded at $500 \mathrm{kbit} / \mathrm{s}$ or $125 \mathrm{kbit} / \mathrm{s}$. The LE coded PHYs are modulated using GFSK at $1 \mathrm{Msym} / \mathrm{s}$ rate, but the payload data are coded in two stages: first by forward error correction convolutional encoder and then spread by the pattern mapper. In theory, this enables to improve the link budget of a coded transmission by over $5 \mathrm{~dB}$ and $12 \mathrm{~dB}$ compared to LE $1 \mathrm{M}$ for $\mathrm{LE}$ coded at $500 \mathrm{kbit} / \mathrm{s}$ and $125 \mathrm{kbit} / \mathrm{s}$ respectively. Note, that only support of LE $1 \mathrm{M}$ PHY is mandatory.

Another change introduced to improve the communications range is the increase of the maximum transmit power of a BLE from
$10 \mathrm{dBm}(10 \mathrm{~mW})$ to $20 \mathrm{dBm}(100 \mathrm{~mW})$. Unfortunately, due to the transmit power restrictions imposed by the frequency regulations, this higher transmit power does not provide any benefit for some regions (namely, EU, Japan and Korea). The maximum link layer protocol data unit (PDU), increased in BLE 4.2 from 39 to 257 octets, stayed at this level also in BLE 5. The problem of coexistence of devices in the $2.4 \mathrm{GHz}$ band has been addressed in BLE 5 by introducing the special interface proving signaling and messaging mechanisms between collocated Bluetooth and other mobile wireless standard radios.

In addition to these changes, the functionality of the broadcasting channels in BLE 5 has been substantially enriched by the introduced extended advertising feature. First, the concept of the secondary advertising channels which are co-allocated with the BLE data channels was introduced. The format of the advertising packets used in the secondary channels has been reworked enabling them to carry up to 255 octets of PDU (compared with 37 octets allowed in the primary channels of BLE 4) and even to support fragmentation. Another interesting feature enabled in the BLE 5 is the periodic advertisements. Hopping between the secondary channels in a predefined pseudo-random sequence, a periodic advertiser broadcasts the packets, with PDU of up to 255 octets, at regular intervals of time ranging from $7.5 \mathrm{~ms}$ to almost $82 \mathrm{~s}$. Importantly, a scanner device may synchronize with one or even several non-overlapping (in time) periodic advertisers and get the data from all of them. This equips BLE 5 with a more efficient and reliable solution for data broadcast than the one possible with BLE 4. Note, that the support of periodic advertisements and extended advertising features is optional.

Importantly, the BLE 5 is backward-compatible with the earlier versions of BLE - all the discussed features are optional and are not necessarily needed to be supported. Nonetheless, as one can easily see, they can substantially increase the communications range or throughput or enable new modes of operation. Due to this fact, in the marketing materials of Bluetooth SIG [13], the BLE 5 is claimed to provide double bandwidth, up to four times higher range and up to 8 times broadcasting capacity compared to BLE 4.2 as summarized in Table 1. However, it must be noted that the improved data rate and communication range cannot be achieved at the same time since they are provided by different PHY modes.

Table 1: Summary of BLE 5 specification characteristics.

\begin{tabular}{|c|c|c|c|c|c|}
\hline PHY & $\begin{array}{l}\text { Error } \\
\text { control }\end{array}$ & $\begin{array}{l}\text { Range } \\
\text { multiplier }\end{array}$ & $\begin{array}{l}\text { PDU } \\
\text { length }\end{array}$ & $\begin{array}{l}\text { Packet } \\
\text { duration }\end{array}$ & $\begin{array}{l}\text { Max. } \\
\text { throughput }\end{array}$ \\
\hline $1 \mathrm{M}$ & $\overline{\mathrm{CRC}}$ & $1 \mathrm{x}$ & $\begin{array}{ll}0 & - \\
257 & B\end{array}$ & $\begin{array}{l}80 \mu \mathrm{s}- \\
2.12 \mathrm{~ms}\end{array}$ & $800 \mathrm{kbps}$ \\
\hline $2 \mathrm{M}$ & CRC & $0.8 \mathrm{x}$ & $\begin{array}{ll}0 \quad- \\
257 \mathrm{~B}\end{array}$ & $\begin{array}{l}44 \mu \mathrm{s}- \\
1.064 \mathrm{~ms}\end{array}$ & $1438 \mathrm{kbps}$ \\
\hline $\begin{array}{l}\text { Coded, } \\
S=2\end{array}$ & $\begin{array}{l}\text { CRC \& } \\
\text { FEC }\end{array}$ & $2 x$ & $\begin{array}{ll}0 \quad- \\
257 \mathrm{~B}\end{array}$ & $\begin{array}{l}462 \mu \mathrm{s}- \\
4.542 \mathrm{~ms}\end{array}$ & $382 \mathrm{kbps}$ \\
\hline $\begin{array}{l}\text { Coded, } \\
S=8\end{array}$ & $\begin{array}{l}\text { CRC \& } \\
\text { FEC }\end{array}$ & $4 x$ & $\begin{array}{l}0 \quad- \\
257 \mathrm{~B}\end{array}$ & $\begin{array}{l}720 \mu \mathrm{s}- \\
17.04 \mathrm{~ms}\end{array}$ & $112 \mathrm{kbps}$ \\
\hline
\end{tabular}




\section{HARDWARE AND SOFTWARE}

In our experiments we have used one of the first commercial chipsets that support BLE 5.0, the nRF52840 [14] from Nordic Semiconductor. The nRF52840 is a system on chip (SoC) integrating a multiprotocol $2.4 \mathrm{GHz}$ transceiver with an ARM Cortex-M4F based microcontroller. The chipset was programmed with S140 v5.0.0 (alpha) SoftDevice, which is a precompiled and linked binary software implementing BLE protocol developed by Nordic Semiconductor. Note that in the time of our experiments S140 was the only SoftDevice providing support for some of the BLE 5 features.

In the experiments we have used two nRF52840 Preview DK development kits shown in Figure 1 with the laptop running a measurement software. The firmware for them was developed in this work based on the ATT_MTU Throughput Example of the nRF5 software development kit (SDK) v13.0.0-1.alpha. Executing this firmware, the two devices first use an $L E I M$ advertising channel to discover each other, establish the connection and set the desired connection parameters (including the used PHY). After the start of the experiment one of the boards, connected to a computer via serial interface, starts spamming the Attribute Protocol (ATT) notifications to the other board at maximum rate. The amount of data sent are periodically reported via the serial interface. Once the pre-defined amount of data bytes has been sent, the experiment is ended and the average throughput is calculated.

Especially for this tests the firmware has been modified. First, we made the boards send data indefinitely while the link is active. Second, we have enabled measurements of the received signal strength indicator (RSSI) by the board with serial interface enabled. Third, we have enabled the control over the transmit power of the board with serial interface enabled. Fourth, we have included in the periodic reports the precise number of bytes sent in notifications and the time passed since the beginning of the experiment, as well as the average RSSI of the last eight radio packets received. These results were logged down and further statistically processed. The first two values have also been later used to calculate the average throughput.

Note, that at a time of our experiments the support for BLE 5 functionality by nRF52840 chipset was limited to enablement of LE $2 M$ and $125 \mathrm{kbit} / \mathrm{s}$ LE Coded PHYs in data channels only. Prior to this, the connection has to be established using LE IM PHY. This limitation has sufficiently affected the procedure of our measurements. Namely, in the initial phase the two devices were placed close enough to each other to enable them establishing the connection using the desired parameters (i.e., maximum transfer unit size, data channel PHY, transmit power, connection interval, etc.). After this, one of the devices (the one receiving the notifications - referred further in the paper as Rx node) was kept static and the other one (Tx node) was moved away from it until the connection was broken. This enabled us to detect the maximum communications range for particular set of parameters. Within this range we have selected few points and measured the average throughput and RSSI in order to investigate how the propagation environment affects the performance of BLE communications.

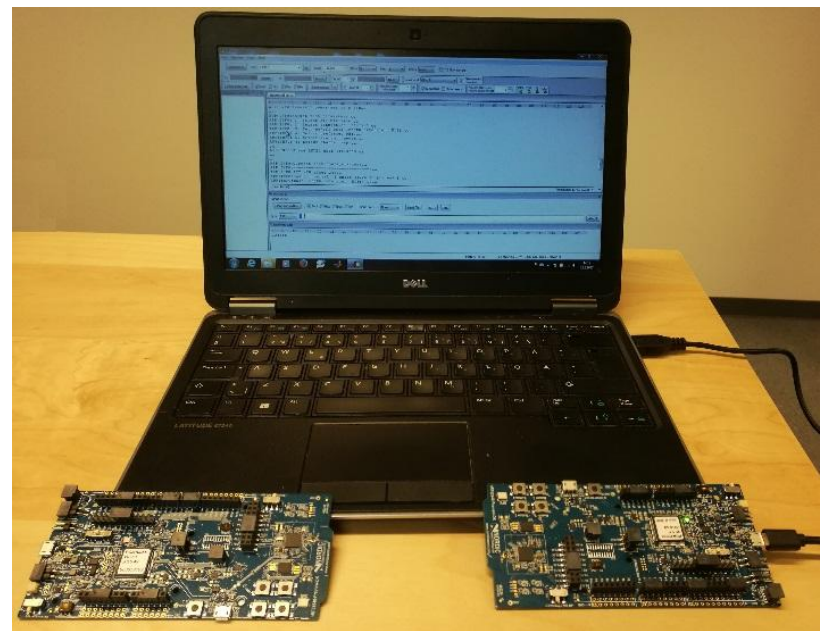

Figure 1: Devices used for measurements.

\section{INDOOR MEASUREMENTS}

The measurements were conducted in the Tietotalo Building of the University of Oulu. The first scenario was on the fourth floor of the north-west wing of the building illustrated in Figure 2. The node receiving the notifications is represented by a red dot.

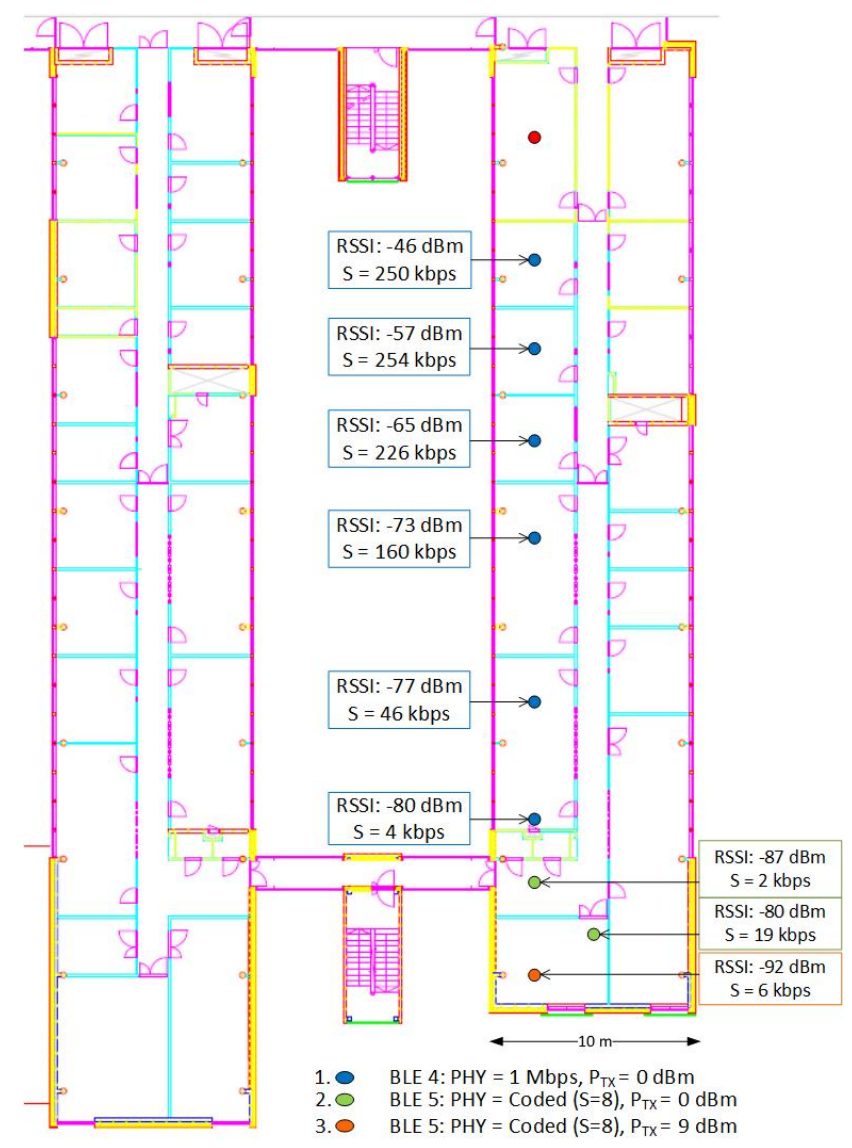

Figure 2: Results of the indoor measurements. 
Blue dots represent the RSSI and throughput values measured for $L E 1 M$ PHY mode using $0 \mathrm{dBm}$ transmit power. The green dots represent the RSSI and throughput values measured when in BLE 5 long-range (125 kbps data) mode, $0 \mathrm{dBm}$ Tx power and $\mathrm{S}=8$ coding were used. The orange dot presents a point for BLE 5 longrange mode with $9 \mathrm{dBm}$ transmit power. Most of the interiors' walls are standard plaster boards except for the bathrooms' walls (represent as green lines) which are made of concrete. The bathrooms and their walls have significant number of metallic pipes and fixtures. We carried out measurements for a plaster wall and concrete wall by using the same devices. It was found out that for a plaster wall the attenuation was only 1-2 $\mathrm{dB}$ and for the concrete wall the attenuation was 4-5 dB.

The second scenario was from a fixed Rx node location in the south-east corner of the fourth floor to the corresponding Tx node location in the third, second, and first floor of the building. In this case, we measured how much is the floor attenuation in this Tietotalo building. This measurement we did at first using BLE 4 PHY with $0 \mathrm{dBm}$ transmit power. The reference RSSI value was $13 \mathrm{dBm}$ when the Tx and Rx node are close to each other. Received RSSI value from 3 rd floor (one floor between Tx and Rx) was -60 $\mathrm{dBm}$ and throughput was $210 \mathrm{kbps}$. Received RSSI value from $2 \mathrm{nd}$ floor (two floors between Tx and Rx) was $-81 \mathrm{dBm}$ and throughput was $40 \mathrm{kbps}$. By using BLE 4 mode we were not able to reach the 1st floor. Therefore we changed to coded $(S=8)$ BLE 5 mode and when using $0 \mathrm{dBm}$ transmit power, we still were not able to reach first floor. With $9 \mathrm{dBm}$ transmit power, the BLE 5 coded mode connection range reach the first floor with RSSI value $-91 \mathrm{dBm}$ and throughput $10 \mathrm{kbps}$.

In these indoor measurements, the communications range improvement that was achieved by using the coded BLE 5 mode is around one extra room between the Tx and Rx nodes. The coding gain was found to be approximately $9 \mathrm{dBm}$. Measurements between different floors showed that by using BLE 4 mode with $0 \mathrm{dBm}$ transmit power, the communication was successful from 4th floor to second floor. In order to reach the first floor, we had to change to BLE 5 coded $(\mathrm{S}=8)$ mode and increase the transmission power. One floor addition to communications range was achieved when using coded mode and increasing the transmission power to $9 \mathrm{dBm}$. We can conclude that when changing from BLE 4 mode to coded BLE 5 mode with same transmit power, the range improvement is around $10 \%$ in this type of indoor office communications. When increasing the transmission power by $9 \mathrm{dBm}$, an additional $10 \%$ improvement can be achieved. The range improvement is not much in meters, but when operating at the communications range limits, the reliability is better when using the coded BLE 5 mode. This is an important factor when a patient carrying wearables is moving inside a hospital and is continuously being monitored.

\section{OUTDOOR MEASUREMENTS}

Outdoor measurements have been conducted for line-of-sight (LOS) and non-line-of-sight (NLOS) scenarios which will be introduced in Sections 5.1 and 5.2, respectively.

\subsection{Line-of-sight case}

The first LOS measurements were conducted outdoors on a bicycle path at Kaitoväylä, Oulu, having a straight line of 640 meters. The purpose of this measurement was to find out the maximum communications range for BLE 4 and BLE 5 with coded PHY when using the maximum transmit power $9 \mathrm{dBm}$, since increase of the communications range is claimed as one of the major design targets of BLE 5. Results of this measurement can be seen on the map of Figure 3. The red dot represent the Rx node location. The blue dots presents measurement points for BLE 4 mode, the final point where connection was broken was found to be at $430 \mathrm{~m}$ distance. Then we changed to BLE 5 coded $(S=8)$ mode and found out that this road was not long enough for the LOS measurements since the connection was still working at the end of bicycle road at 640 meters distance between Tx and Rx node. However, the results are presented here to complement the other outdoor LOS measurement that are described below. The green dot represent the measurement point and results for BLE 5 coded mode in this scenario.

The second outdoor LOS measurements were conducted on a longer straight $(\sim 1 \mathrm{~km})$ bicycle road to find out the maximum communications distance also for BLE 5 coded mode. In order to complement the set of previous measurements, we measured at first BLE 4 and BLE 5 coded mode performances using transmit power

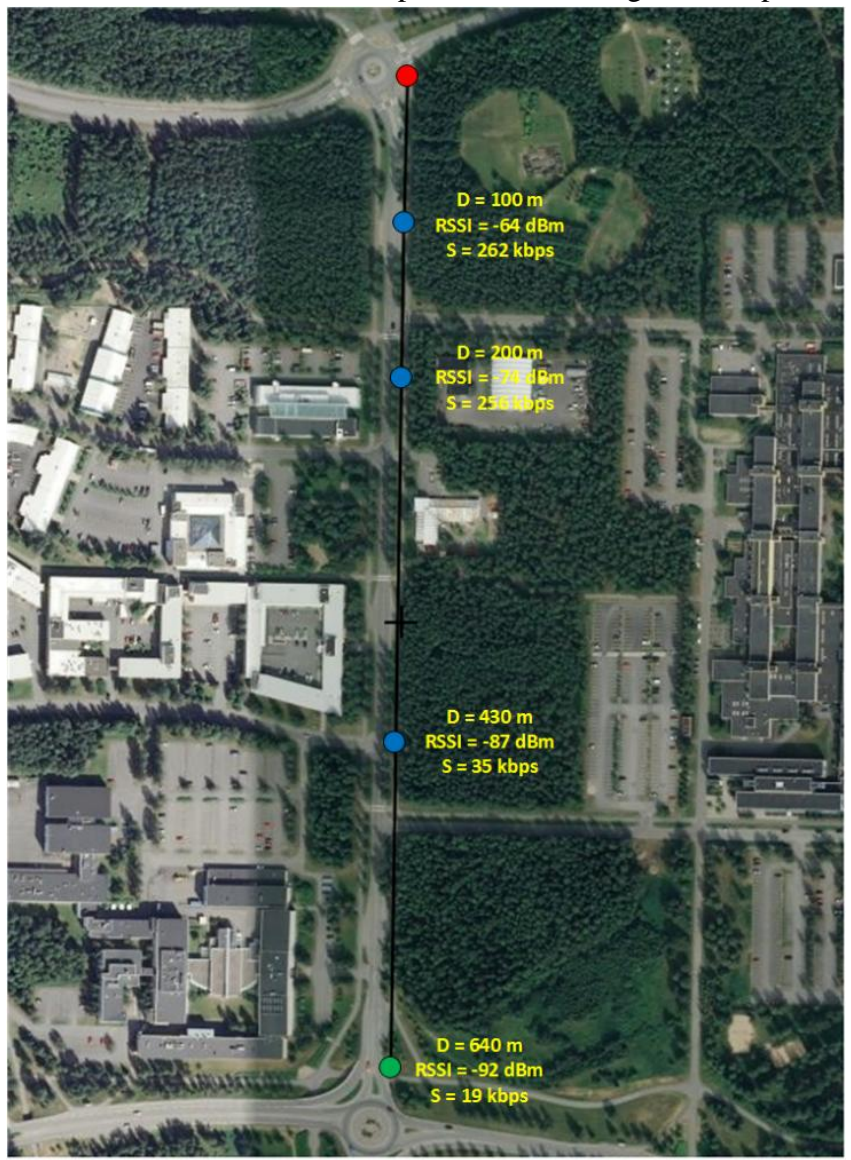

Figure 3: Results of the first outdoors LOS measurements. 
of $0 \mathrm{dBm}$ and then used the BLE 5 mode with transmit power of 9 $\mathrm{dBm}$. This very long straight bicycle path allowed for LOS measurement, as illustrated in Figure 4 taken from the Rx point.

Results are shown for each case in Figure 5. The RSSI and throughput values just before connection break are shown for each case. Red circle is the RX point and blue circle is the point where BLE 4 connection was broken. Yellow circle is the point where BLE 5 mode with transmit power $0 \mathrm{dBm}$ stopped working, and green circle is the point where connection was broken when using BLE 5 mode with transmit power of $9 \mathrm{dBm}$. From the results of Figure 5 it can be observed that for BLE 4 with $0 \mathrm{dBm}$, the maximum range was $220 \mathrm{~m}$. For BLE 5 case with $0 \mathrm{dBm}$ transmit power, the communications range was found to be $490 \mathrm{~m}$. In the previous outdoor measurements it was found that for BLE 4 with 9 $\mathrm{dBm}$, the maximum range was $430 \mathrm{~m}$. Therefore, the BLE 5 coded mode can achieve longer range with $9 \mathrm{~dB}$ lower transmit power $(0$ $\mathrm{dBm})$. Figure 5 shows that the communications range for BLE 5 coded $(\mathrm{S}=8)$ mode with transmit power of $9 \mathrm{dBm}$ is very impressive, being 780 meters.

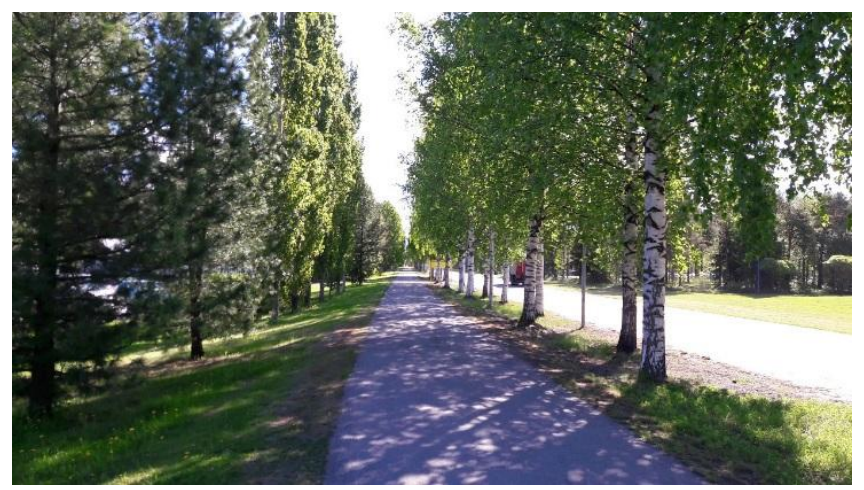

Figure 4: Straight bicycle road used for LOS measurements.

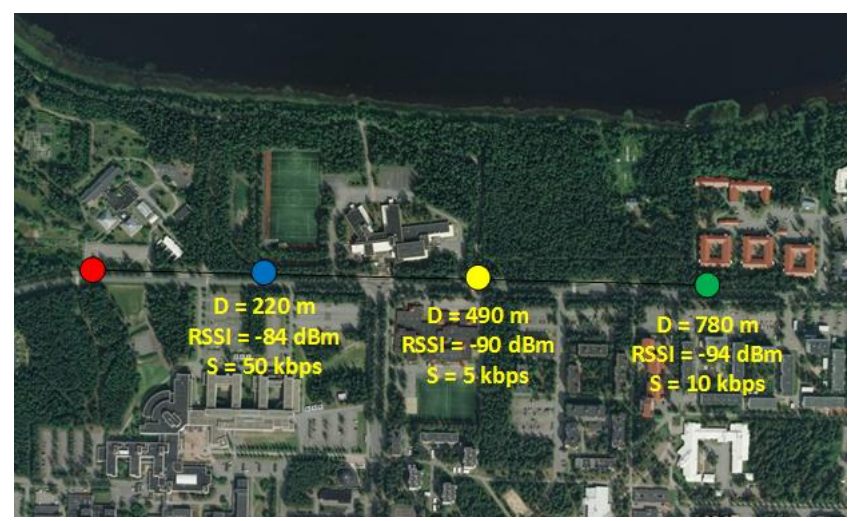

Figure 5: Results of the second outdoors LOS measurements.

\subsection{Non-line-of-sight case}

We did measurements at University of Oulu Botanical Garden to find out the communications range of BLE 4 and BLE 5 in the case of non-line-sight situation where trees and bushes block the signal. Measurements were done in two different scenarios
('Scen1' and 'Scen2') with different type of obstructions created by vegetation.

The first measurement set (Scen1) was done by setting the $\mathrm{Rx}$ node on top of small hill (approx. $5 \mathrm{~m}$ height) and moving the Tx node around the garden to find out when the connection breaks in case of BLE 4 and BLE 5 coded mode. A view from the Rx node position is shown in Figure 6 to illustrate the type of vegetation of the measurement environment. The walking route and connection breaking points for this measurement set are shown in Figure 7. In this scenario the transmit power was set to $9 \mathrm{dBm}$ for both BLE 4 and BLE 5 coded mode.

In BLE 4 case the connection was broken at the blue circle point shown in Figure 7. Connection quality was good via the shown walking path at the garden, average being $190 \mathrm{kbps}$, even there are trees and bushes blocking the signal between Tx and Rx nodes. The final measured RSSI value before the connection break was -88 $\mathrm{dBm}$ and throughput was $2 \mathrm{kbps}$.

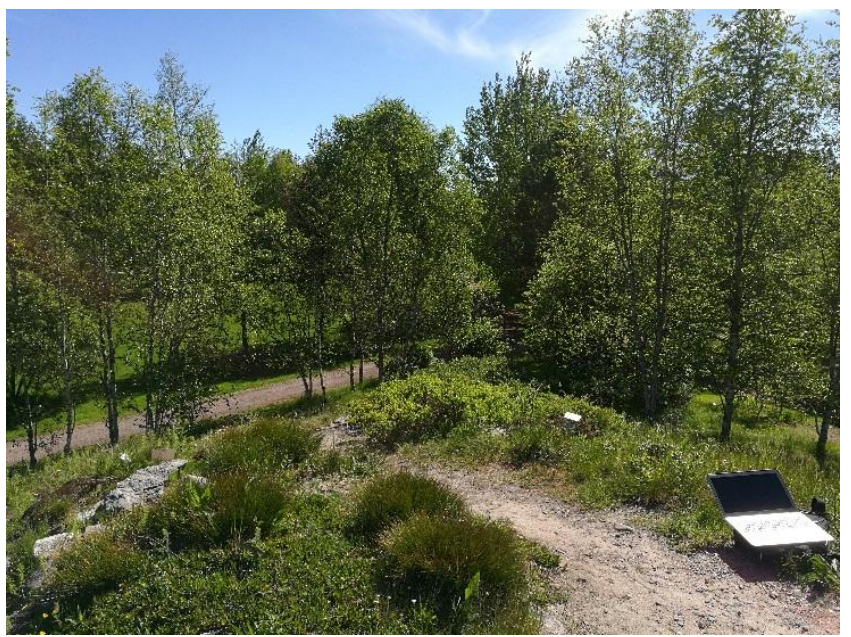

Figure 6: View from the Rx node position in NLOS measurement Scen1.

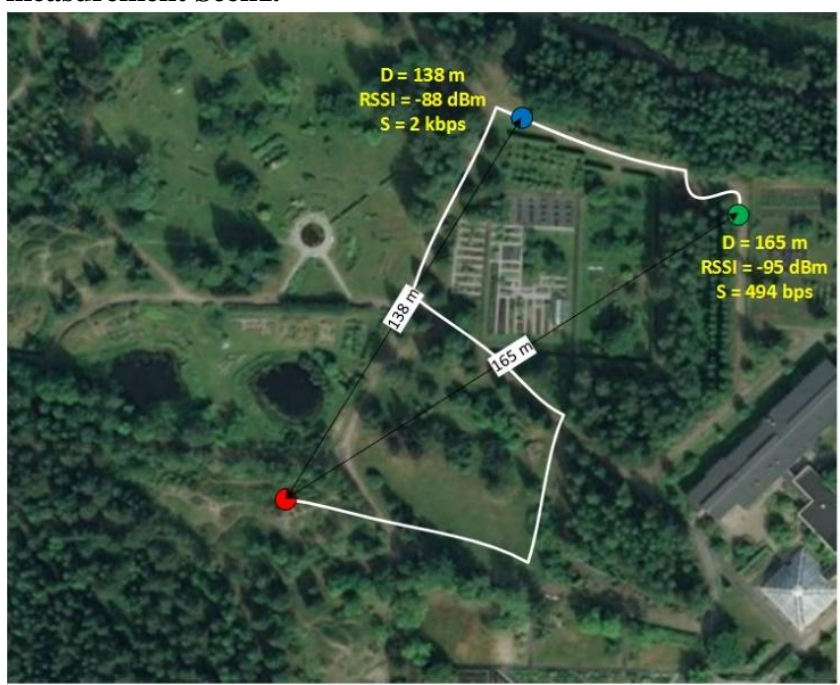

Figure 7: Walking path and results of NLOS Scen1. 
In BLE 5 case, the connection was working longer, as expected, and was broken at a point marked with green circle in Figure 7. However, the range improvement is not drastic in this NLOS scenario. The NLOS path was very difficult at the point where BLE 5 connection was broken since there was a dense tree stand. The final measured RSSI value before connection break was $-95 \mathrm{dBm}$ and throughput was 494 bps. This behavior is somewhat similar to the NLOS indoor case since the relative range improvement is not very large.

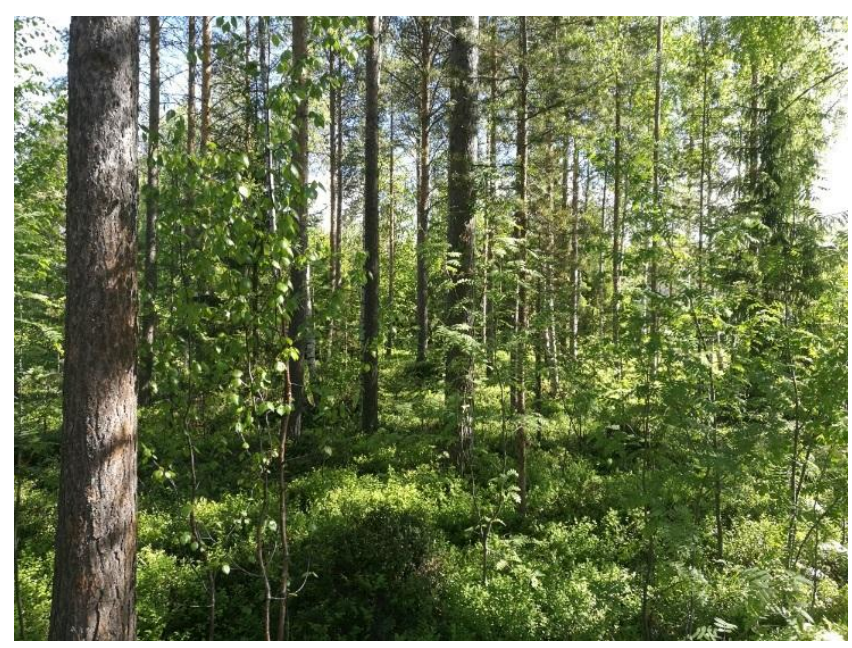

Figure 8: View from the Rx node position in NLOS measurement Scen2.

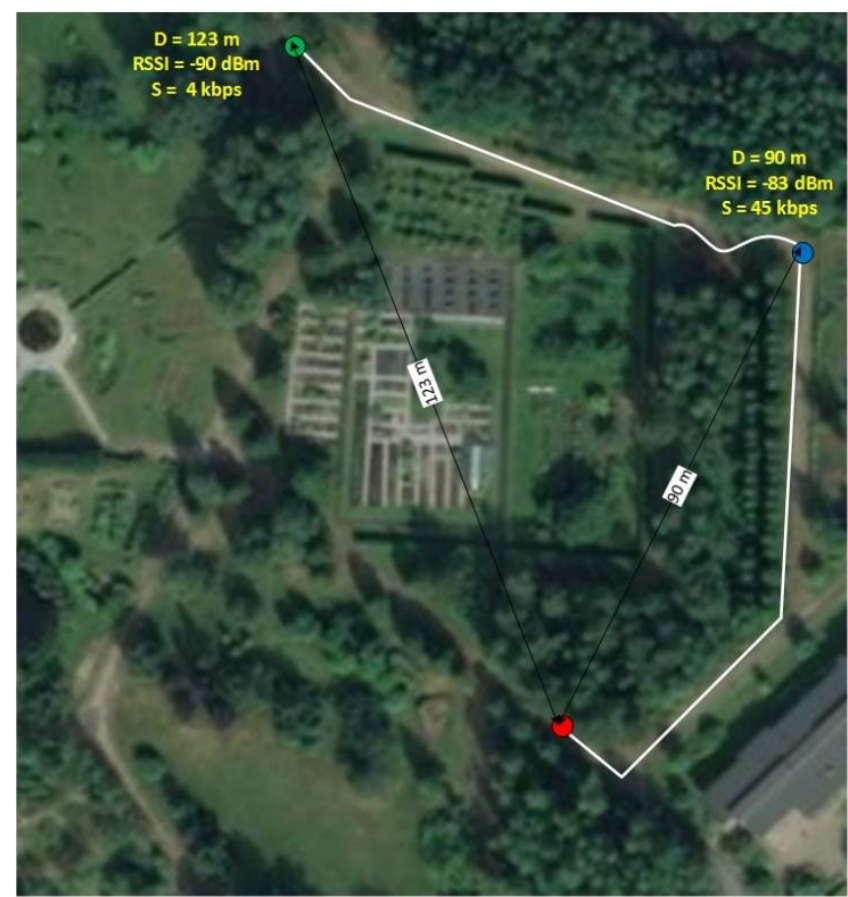

Figure 9: Walking path and results of NLOS Scen2.

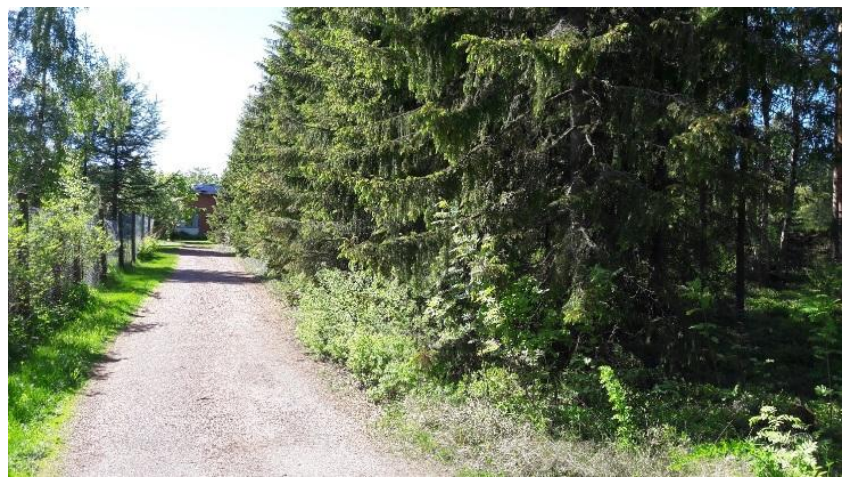

Figure 10: Vegetation at the point where BLE 4 connection was broken in NLOS Scen2.

In the second outdoor NLOS scenario (Scen2) the Rx node was $1 \mathrm{~m}$ above the ground level and the vegetation was dense as illustrated in Figure 8. The walking path and results are illustrated in Figure 9. In BLE 4 case, the connection was broken at the point which is shown in Figure 9 by using a blue circle. We can see from the result that the achievable communications range through a heavy vegetation was $90 \mathrm{~m}$. Average throughput for this walking path measurement was $170 \mathrm{kbps}$. Vegetation at the end point of this case is shown in Figure 10 and from the starting point towards the end point is illustrated in Figure 8. Then BLE 5 coded mode was measured using the same transmit power of $0 \mathrm{dBm}$. The point where the connection was broken is shown using a green circle in Figure 9. We can see that this mode was able to increase the communications distance to 123 meters. However, it must be noted that the vegetation is not homogenous through the measurement path as can be seen from the Figure 9.

Outdoor measurements illustrate the improved communications distance when using BLE 5 coded mode especially in the LOS case. The coding gain was found to be approximately $9 \mathrm{dBm}$. We can conclude also that in general, BLE performance is quite good even with quite heavy vegetation at the communication path. When there is a heavy vegetation, the range improvement (BLE 4 vs BLE 5) behavior is somewhat similar to indoor case, i.e., the relative improvement in distance is not drastic. BLE 5 coded $(S=8)$ mode with transmit power of $9 \mathrm{dBm}$ enables a very long communications range in LOS conditions, being $780 \mathrm{~m}$ in our real-life measurements.

\section{DISCUSSION OF THE RESULTS}

Based on the introduced indoor and outdoor results, which are summarized also in Table 2, we can compare the communications range performance of BLE 4 vs BLE 5 coded version. It is evident that BLE 5 coded version can improve the communications range, in comparison to BLE 4 version, which has been its design goal. However, based on our measurement results it can be seen that most of the time the range improvement is not 4-fold as has been advertised in BLE 5 marketing material. Based on the results introduced in this paper, and our additional measurement experiments with the same development kit, we can conclude that the gain of the coded mode is approximately $9 \mathrm{~dB}$. Namely, in the 
indoor and outdoor measurement we have found that the BLE 5 coded mode can achieve approximately similar communications range than BLE 4 mode, by using $9 \mathrm{~dB}$ lower transmit power. When using the same transmit power for both modes, the range improvement has been found to be around $10-20 \%$ for indoor scenario. In NLOS outdoor scenario, the range improvement was found to be approximately $20-37 \%$ in our measurement scenarios. However, it must be noted that in outdoor scenarios the vegetation was not homogenous, as well as in indoor scenarios the construction material is varying depending on the signal path, therefore in order to acquire exact range improvement percentages and coding gain, more measurement are needed to find out statistically reliable results. Outdoor LOS measurements showed that the BLE 5 coded mode can improve the communications range more than 2-fold when using the same transmit power than BLE 4 mode. When using the transmit power of $9 \mathrm{dBm}$, the communications range of BLE 5 coded mode was found to be very impressive, 780 meters, which is closer to 4 -fold improvement in comparison to BLE 4 range with $0 \mathrm{dBm}$ transmit power. Therefore, our finding is that the 4-fold communications range improvement requires the BLE 5 coded mode combined with increased transmit power.

Table 2: Summary of measurement results.

\begin{tabular}{|c|c|c|c|c|}
\hline Mode & $\begin{array}{l}\text { Tx } \\
\text { Power }\end{array}$ & Range & $\begin{array}{l}\text { Max } \\
\text { Throughput }\end{array}$ & $\begin{array}{l}\text { Min } \\
\text { Throughput }\end{array}$ \\
\hline \multicolumn{5}{|c|}{ Indoor Scenario } \\
\hline BLE 4 & $0 \mathrm{dBm}$ & $43 \mathrm{~m}$ & $262 \mathrm{kbps}$ & $1761 \mathrm{bps}$ \\
\hline BLE 5 & $0 \mathrm{dBm}$ & $48 \mathrm{~m}$ & $26 \mathrm{kbps}$ & $521 \mathrm{bps}$ \\
\hline BLE 5 & $9 \mathrm{dBm}$ & $51 \mathrm{~m}$ & $26 \mathrm{kbps}$ & $3.5 \mathrm{kbps}$ \\
\hline \multicolumn{5}{|c|}{ Outdoor NLOS Scen1 } \\
\hline BLE 4 & $9 \mathrm{dBm}$ & $138 \mathrm{~m}$ & $262 \mathrm{kbps}$ & $2.7 \mathrm{kbps}$ \\
\hline BLE 5 & $9 \mathrm{dBm}$ & $165 \mathrm{~m}$ & $26 \mathrm{kbps}$ & 494 bps \\
\hline \multicolumn{5}{|c|}{ Outdoor NLOS Scen2 } \\
\hline BLE 4 & $0 \mathrm{dBm}$ & $90 \mathrm{~m}$ & $262 \mathrm{kbps}$ & $8.3 \mathrm{kbps}$ \\
\hline BLE 5 & $0 \mathrm{dBm}$ & $123 \mathrm{~m}$ & $26 \mathrm{kbps}$ & $2.1 \mathrm{kbps}$ \\
\hline \multicolumn{5}{|c|}{ Outdoor LOS } \\
\hline BLE 4 & $0 \mathrm{dBm}$ & 220 & $262 \mathrm{kbps}$ & $1.1 \mathrm{kbps}$ \\
\hline BLE 4 & $9 \mathrm{dBm}$ & 430 & $262 \mathrm{kbps}$ & $7.5 \mathrm{kbps}$ \\
\hline BLE 5 & $0 \mathrm{dBm}$ & 490 & $26 \mathrm{kbps}$ & $1.7 \mathrm{kbps}$ \\
\hline BLE 5 & $9 \mathrm{dBm}$ & 780 & $26 \mathrm{kbps}$ & $1.1 \mathrm{kbps}$ \\
\hline
\end{tabular}

\section{CONCLUSIONS AND FUTURE WORK}

This paper described experimental communications range and throughput measurement results for new the BLE 5 coded mode. Reference measurements are also conducted using BLE 4 (1 Mbps) PHY settings. The goal was to find out the performance of the BLE 5 coded $(S=8)$ version, since with improved communications range it can open up more options and enable new uses for BLE in medical / healthcare scenarios. Results show that BLE 5 coded mode improves the communications range, with lower throughputs that are acceptable for low-power sensor devices. However, our measurements show that the promised 4-fold communications range improvement for BLE 5 requires both coded mode and increased transmission power. The coding gain of BLE 5 coded version $(S=8)$ was found to be approximately $9 \mathrm{~dB}$, i.e., the coded version was able to achieve same (or longer) communications range than BLE 4 by using $9 \mathrm{~dB}$ lower transmission power. The maximum BLE 5 communications range which we have witnessed in our experiments outdoors was almost 800 meters, using the transmit power of $9 \mathrm{dBm}$. The presented results indicate that BLE 5 is an efficient technology that can be used in remote patient monitoring via wireless connectivity between wearable nodes carried by the mobile patients. Larger coverage is accomplished by using strong coding which reduces the amount of required infrastructure at the hospital buildings.

To the best of our knowledge, this paper reports the first measurement campaign conducted to find out the performance of BLE 5 coded mode and having it compared to BLE 4.2. In future work, we plan to perform additional measurements to study the effect of different PHY settings that the new BLE 5 version offers compared to previous BLE version. Specifically, since the increase of the range by using the coded PHY brings along the increase of the on-air time, we plan to study the interference nature of other wireless technologies on BLE 5. In addition, we plan to perform measurements around the human body since it is a difficult communications environment.

\section{ACKNOWLEDGMENTS}

This work has been partially funded by the European Regional Development Fund (ERDF) through the WILLE project.

\section{REFERENCES}

[1] P. Lamkin, "Wearable Tech Market To Be Worth \$34 Billion By 2020," Feb. 2016, https://www.forbes.com/sites/paullamkin/2016/02/17/wearable-techmarket-to-be-worth-34-billion-by-2020/\#4cbe76e83cb5.

[2] Tractica, "Healthcare Wearable Device Shipments to Reach 98 Million Units Annually by 2021," April 2016,

https://www.tractica.com/newsroom/press-releases/healthcare-wearabledevice-shipments-to-reach-98-million-units-annually-by-2021/.

[3] H. Karvonen, M. Hämäläinen, J. Iinatti and C. Pomalaza-Ráez, "Coexistence of Wireless Technologies in Medical Scenarios," European Conference on Networks and Communications (EUCNC), Oulu, Finland, June 2017.

[4] IEEE 802.11 LAN Working Group, http://www.ieee802.org/11/.

[5] IEEE Standard for Low-Rate Wireless Networks,"IEEE Std 802.15.4-2015 (Revision of IEEE Std 802.15.4-2011)” April 22, 2016.

[6] ZigBee Alliance, http://www.zigbee.org/.

[7] IEEE Std. 802.15.6: IEEE Standard for Local and metropolitan area networks-Part 15.6: Wireless Body Area Networks. Standard, The Institute of Electrical and Electronics Engineers, Inc., 2012.

[8] M. Hämäläinen et al., "ETSI TC SmartBAN: Overview of the wireless body area network standard," International Symposium on Medical Information and Communication Technology (ISMICT), 2015.

[9] ANT/ANT+, https://www.thisisant.com/.

[10] Nokia white paper, "LTE evolution for IoT connectivity," 2017.

[11] H. Karvonen, C. Pomalaza-Ráez, K. Mikhaylov, M. Hämäläinen and J. Iinatti, "Interference of Wireless Technologies on BLE Based WBANs in Hospital Scenarios," IEEE International Symposium on Personal, Indoor and Mobile Radio Communications (PIMRC), Montreal, Canada, October 2017.

[12] Bluetooth SIG, "Bluetooth Core Specification v 5.0" https://www.bluetooth.com/specifications/bluetooth-core-specification, December 2016.

[13] Bluetooth SIG, "Bluetooth Core Specification 5.0 FAQ" https://www.bluetooth.com/ /media/files/specification/bluetooth-5faq.ashx?la=en, 2016.

[14] Nordic nRF52840, https://www.nordicsemi.com/eng/Products/nRF52840. 